\section{EFSUMB at ECR 2017}

At ECR 2017, the joint ESR/EFSUMB session 'Hand held devices - Game changer?' included a summary of EFSUMB's position on medical student education using ultrasound. Vito Cantisani (Chairman of the EFSUMB EPSC) suggested that although education for students is traditionally based on training methods such as presentations, courses and workshops, the new technologies such as ultrasound equipment and web-based information have new educational applications.

Ultrasound is recognised as an effective tool in anatomic and pathologic evaluation of different organs. In addition, availability of new ultrasound scanners, portable and miniaturized, with lower costs and greater effectiveness offer more teaching possibilities.

Increasingly ultrasound is being used, not just by radiologists but also by a broad range of clinical specialists. With competency it offers enhanced opportunities for diagnosis and treatment. The need for qualitative standardization of minimum competency in ultrasound before being allowed to practice was raised in the session discussion. Throughout Europe there is differentiation in competency levels (basic, advanced, teacher), which should be defined based on modules throughout Europe.

In the last few years, US has been proved to be effective for diagnostic clinical purposes increasing the quality of clinicians' diagnosis and management in almost all areas of medicine. Experience in the USA, at Har- vard and North Carolina Universities and in Europe at Oslo, Bergen, Gdansk and Vienna suggest that US can be effective for educational purposes. EFSUMB decided to focus on student education in order to promote a standardized introduction in the US teaching programme at the beginning of student training. This is based on the technological innovations such as simulators and hand-held devices. After reviewing the literature, current experience, and preparing educational material, such as the EFSUMB Course Book for students and website material EFSUMB published a position paper in two journals, Ultraschall (short version) and UIO (long version). Additionally a new Student Committee was established in 2016 and a Task Force Group prepared a survey which was sent to many European medical school deans. Only 45 responses from 14 countries have been received which have been evaluated. This survey will be available on the EFSUMB website and sent to all EFSUMB National Societies and mailing list members for completion: https://de.surveymonkey.com/r/897RC8X

From the initial responses we learnt that implementing US into the medical school curriculum has been considered beneficial for the following reasons: ultrasound knowledge is useful for all physicians; it is useful to learn for later clinical practice, it may help students learn anatomy. However, barriers for a better standard US curriculum in medical schools that emerged were: Lack of time (in the curriculum); problems in equipment funding and lack of faculty funding.
Therefore, the three main challenges are:

- US machines (need for investment with help from US producers?) "each student should have a US machine in the pocket";

- SIMULATORS, simple, easy to manipulate, realistic, but expensive and not widely available;

- TEACHERS specialized in medical education and US practitioners in different clinical specialties currently are insufficient to teach US a in an easy way, to explain artefacts and how to avoid them and to explain the limits of the procedure.

Currently, US education of medical students is mostly covered by radiology; however, in many medical schools, other departments, (anatomy, internal medicine, obstetrics and gynaecology urology, etc.) are probably increasingly sharing the load to achieve this educational goal, but not yet in sufficient student education departments. More teachers and hands-on training is the goal.

Deans, medical education departments, other academics and students are aware of the need to implement practical US education into the curriculum since they consider ultrasound as a very good educational tool for medical students. Teachers should be qualified experts to help students to identify the relevant data from the large amounts of information in the US image. Medical School pilot studies results will help to promote proposals for a European curriculum platform.

Vito Cantisani, on behalf of the EFSUMB EPSC and TFG 